\title{
Impairment of the retinoic acid-inducible gene-I-IFN- $\beta$ signaling pathway in chronic hepatitis $B$ virus infection
}

\author{
GANGDE ZHAO $^{1}$, BAOYAN AN ${ }^{1}$, HUIJUAN ZHOU ${ }^{1}$, HUI WANG $^{1}$, YUMIN XU ${ }^{1}$, XIAOGANG XIANG ${ }^{1}$, \\ ZHIXIA DONG ${ }^{1}$, FANGMEI AN ${ }^{1}$, DONGSHAN YU ${ }^{1}$, WEIJING WANG ${ }^{1}$, SHISAN BAO $^{2}$ and QING XIE $^{1}$ \\ ${ }^{1}$ Department of Infectious Diseases, Ruijin Hospital, Shanghai Jiaotong University School \\ of Medicine, Shanghai 200025, P.R. China; ${ }^{2}$ Discipline of Pathology, School of Medical \\ Sciences and Bosch Institute, University of Sydney, Sydney, NSW 2006, Australia
}

Received April 29, 2012; Accepted July 13, 2012

DOI: 10.3892/ijmm.2012.1131

\begin{abstract}
Chronic hepatitis B (CHB) virus infection is caused by compromised host immunity, but the precise underlying mechanism remains unclear. Retinoic acid-inducible gene I (RIG-I) triggers antiviral immunity by inducing interferon- $\beta$ (IFN- $\beta$ ) production following viral infection. To investigate the role of the RIG-I-IFN- $\beta$ signaling pathway in monocytederived dendritic cells (moDCs) during CHB infection, moDCs were generated by stimulating $\mathrm{CD} 14^{+}$monocytes in vitro. MoDCs from patients with CHB, acute hepatitis B (AHB) and healthy controls $(\mathrm{HCs})$ were challenged with vesicular stomatitis virus (VSV) and the levels of RIG-I, IFN- $\beta$ promoter stimulator 1 (IPS-1) and IFN- $\beta$ in the stimulated moDCs were determined. Following $16 \mathrm{~h}$ of VSV stimulation, RIG-I expression was reduced by $50 \%$ in moDCs from CHB patients and by $70 \%$ in moDCs from AHB patients relative to $\mathrm{HC}$ moDCs, concomitant with a $20 \%$ decrease in IFN- $\beta$ expression in CHB patients relative to AHB patients and HCs. Additionally, a significant correlation between the RIG-I/IPS-1 ratio and alanine aminotransferase (ALT) level was observed. To further investigate the function of RIG-I in chronic hepatitis B virus
\end{abstract}

Correspondence to: Professor Qing Xie, Department of Infectious Diseases, Ruijin Hospital, Shanghai Jiaotong University School of Medicine, 197 Ruijin Er Road, Shanghai 200025, P.R. China

E-mail: xieqingrj@gmail.com

Professor Shisan Bao, Discipline of Pathology (D06), School of Medical Sciences and Bosch Institute, University of Sydney, Sydney, NSW 2006, Australia

E-mail: bob.bao@sydney.edu.au

Abbreviations: AHB, acute hepatitis B; ALT, alanine aminotransferase; CARD, caspase recruitment domain; $\mathrm{CHB}$, chronic hepatitis $\mathrm{B}$; HC, healthy control; IFN- $\beta$, interferon- $\beta$; IPS- 1 , IFN- $\beta$ promoter stimulator 1; MDA-5, melanoma differentiation-associated gene 5; moDC, monocyte-derived dendritic cell; PBMC, peripheral blood mononuclear cell; RIG-I, retinoic acid-inducible gene I; VSV, vesicular stomatitis virus

Key words: chronic hepatitis B, dendritic cell, retinoic acid-inducible gene I, interferon, innate immunity
(HBV) infection, HepG2 or HepG2.2.15 (HBV-transformed) cell lines were challenged with VSV following RIG-1 transfection. IFN- $\beta$ induction was suppressed in HepG2.2.15 cells, but was restored following RIG-I transfection. Taken together, these data indicate that compromised moDC function in $\mathrm{CHB}$ patients is attributable to an impaired RIG-I-IFN- $\beta$ signaling pathway, which results in compromised host viral clearance and HBV persistence in a susceptible population.

\section{Introduction}

Hepatitis B virus (HBV) infection remains a global health concern and is associated with a great economic burden, affecting approximately 400 million people worldwide despite decades of research. HBV infection leads to a broad spectrum of clinical manifestations, including cirrhosis, fulminant hepatic failure and hepatocellular carcinoma (1). Almost $20 \%$ of chronic HBV patients (approximately 2.6 million) in China alone developed cirrhosis and hepatocellular carcinoma. Chronic HBV infection is a consequence of complex interactions between the replicating noncytopathic virus and the host immune system, which plays a critical role in viral clearance. Deficient host immunity subsequently augments the chronic status (2).

Host antiviral innate immunity is initiated via viral pathogen-associated molecular patterns (PAMP), such as viral double-stranded RNA (dsRNA). All RNA and most DNA viruses, including HBV (3), generate dsRNA molecules at the transcription or replication level $(4,5)$. Retinoic acid-inducible gene I (RIG-I), a cytoplasmic RNA helicase, plays an essential role in host antiviral immunity by activating type-I interferon $(\operatorname{IFN}-\alpha / \beta)(6,7)$ in response to intracellular viral RNA recognition. The RIG-I antiviral response is cell-type specific (8) and is enhanced by type-I interferon following an interaction between TLR7-9 and their ligands (9). RIG-I mediated antiviral immunity and its downstream signaling pathway have been well illustrated in HCV infection (10). Interferon- $\beta$ (IFN- $\beta$ ) production is suppressed in the liver following viral invasion, which directly represses RIG-I and its downstream adaptor IFN- $\beta$ promoter stimulator 1 (IPS-1) (11). However, it is unclear whether the RIG-I-mediated signaling pathway contributes to host antiviral immunity in 
HBV. In this study, we investigated the correlation between RIG-I expression and downstream signaling in monocytederived dendritic cells (moDCs) from $\mathrm{HBV}$-infected patients as well as in HBV-transformed cell lines in response to vesicular stomatitis virus (VSV) challenge. This insight may lead to basic research and/or clinical treatment of this disease.

\section{Materials and methods}

$H B V$ patients and healthy subjects. Chronic hepatitis $\mathrm{B}$ patients (CHB; $n=21)$ and acute hepatitis $B$ patients (AHB; $n=7$ ) were recruited for the current study with the following criteria: all patients were $\mathrm{HBV}$ surface antigen ( $\mathrm{HBsAg}$ ) positive but negative for other viruses (e.g., HCV, HDV and HIV) and treatment-naïve for 6 months prior to sample collection. Age-, gender- and ethnic background-matched HBsAg-negative healthy controls ( $\mathrm{HCs} ; \mathrm{n}=18$ ) were selected. The clinical characteristics of patients are listed in Table I. Informed consent was obtained from all subjects. All experimental procedures adhered to the guidelines of the Helsinki Declaration of 1975 and were approved by the Human Ethics Committee, Shanghai Jiaotong University School of Medicine.

Virological assessment. Serum HBsAg, $\mathrm{HBeAg}$ and anti-HBe were determined using commercial enzyme immunoassay kits (AXSYM System; Abbott, Wiesbaden, Germany). HBV DNA levels were quantified by the Applied Biosystems PCR system (Prism 7500; Applied Biosystems, Inc., USA) with the lowest detection limit at 1000 copies/ml as previously described $(12,13)$.

Monocyte collection and moDC induction. Peripheral blood mononuclear cells (PBMC) were isolated from $20 \mathrm{ml}$ of heparinized peripheral blood by Ficoll-Hypaque gradient centrifugation. Monocytes were obtained using a MACS CD14 isolation kit (Miltenyi Biotec, Bergisch Gladbach, Germany) and $>90 \%$ purity was confirmed with flow cytometry. The purified monocytes were seeded in 6-well plates $\left(1.5 \times 10^{6}\right.$ cells $\left./ \mathrm{ml}\right)$ in $2 \mathrm{ml}$ RPMI-1640 supplemented with $10 \%$ heat-inactivated $10 \%$ fetal calf serum, $2 \mathrm{mmol} / \mathrm{l}$ L-glutamine, $100 \mathrm{U} / \mathrm{ml}$ penicillin and $100 \mu \mathrm{g} / \mathrm{ml}$ streptomycin. moDCs were generated by adding GM-CSF (500 U/ml) and IL-4 (250 U/ml) (Peprotech, Inc., Rocky Hill, NJ, USA) in the culture medium for 7 days as previously described (14). The moDCs obtained were challenged with wild-type VSV (15) at a multiplicity of infection (MOI) of 0.1 for activation of RIG-I-mediated signaling.

RIG-I transfection and VSV challenge. VSV was propagated in Wish cells and quantified by standard plaque assay. HepG2 (hepatoma cell line) and HepG2.2.15 cells (hepatoma cell line transformed with HBV) were transfected with RIG-I plasmids (16) by Lipofectamine ${ }^{\mathrm{TM}} 2000$ (Invitrogen Life $^{\mathrm{M}}$ Technologies, Shanghai, China) in DMEM culture medium for $24 \mathrm{~h}$, following the manufacturer's instructions. The RIG-I-transfected cells were cultured with fully supplemented growth medium for $0,8,16$ and $24 \mathrm{~h}$, after a half-hour challenge with VSV (17) at $0.1 \mathrm{MOI}$ in free serum medium. IFN- $\beta$ production in the challenged cells and supernatants was determined by qRT-PCR and ELISA.
RNA isolation, reverse transcription and quantitative realtime PCR. RNA was extracted with an RNAprep pure Micro kit (Tiangen Biotech Co., Ltd., Beijing, China). Total-RNA $(0.5 \mu \mathrm{g})$ was reverse transcribed with the SuperScript first-strand synthesis system for RT-PCR (Invitrogen Life Technologies). RNA expression was quantified by SYBRGreen-based qRT-PCR using a sequence detection system (Prism 7500; Applied Biosystems, Inc.) with GAPDH serving as an endogenous control. The sequences of gene-specific primers are listed in Table II. The threshold cycle $(\mathrm{Ct})$ for each gene was determined as the cycle number at which the reaction crossed an arbitrarily placed threshold, and the amount of each target gene relative to GAPDH was described using the formula $2^{-\Delta \mathrm{Ct}}$ where $\Delta \mathrm{Ct}=\left(\mathrm{Ct}_{\text {target gene }}-\mathrm{Ct}_{\mathrm{GAPDH}}\right)$.

Western blot analysis. HepG2 and HepG2.2.15 cells with different treatments were homogenized in RIPA buffer (20 mM Tris, pH 7.4, 2.5 mM EDTA, 1\% Triton X-100, $10 \%$ glycerol, $1 \%$ deoxycholic acid, $0.1 \%$ SDS, 1 mM PMSF, $10 \mathrm{ng} / \mathrm{ml}$ aprotinin). Western blot analyses were performed as previously described (18). Briefly, extracted protein $(50 \mu \mathrm{g})$ was resolved by SDS-PAGE (10\% gel) and transferred to nitrocellulose membranes. The blots were blocked and labeled with primary antibodies [rabbit anti-human RIG-I, 1:2,000 (Biodesign, USA); or mouse anti-human $\beta$-actin 1:5,000 (Sigma, USA)] overnight. Membranes were incubated with the secondary antibody (goat anti-rabbit-HRP or goat anti-mouseHRP, 1:5,000 each; Amersham Pharmacia Biotech, Saclay, France). The labeled proteins were detected using the ECL Plus kit (Amersham Pharmacia Biotech).

Enzyme-linked immunosorbent assay. The HepG2 and HepG2.2.15 cell supernatant IFN- $\beta$ concentrations were determined using an ELISA kit (R\&D Systems, Minneapolis, MN, USA) at different time points following various treatments.

Statistical analysis. All data are expressed as the mean \pm standard deviation (SD). Comparisons between the 2 groups were made using the Student's t-test. Non-parametric statistical analysis was performed using the Mann-Whitney U-test between 2 independent groups with SPSS 13.0 for Windows (SPSS, Inc., Chicago, IL, USA). Spearman's rho was used for correlation analysis. Differences were considered statistically significant at $\mathrm{P}<0.05$.

\section{Results}

Reduced maturity of moDCs in $H B V$ patients. Mature $\mathrm{CD} 6^{+}$moDCs derived from the VSV-stimulated and GM-CSF/IL-4-stimulated CD14 ${ }^{+}$monocytes were confirmed by flow cytometry (Fig. 1). The proportion of $\mathrm{CD}^{2} 6^{+}$ moDC cells from CHB patients was $17 \%$ lower than in $\mathrm{HCs}$ $(80.77 \pm 8.51$ vs. $96.89 \pm 2.12 \%, \mathrm{P}<0.05)$.

Expression of RIG-I, IPS-1 and IFN- $\beta$ mRNA in the moDC. The expression of RIG-1 mRNA was elevated $\sim 9$-fold at $8 \mathrm{~h}$ post-VSV stimulation in all 3 groups $(\mathrm{CHB}, \mathrm{AHB}$ and $\mathrm{HC}$; Fig. 2) compared to their unchallenged counterparts. No significant difference was observed among these 3 groups in the absence of stimulation. RIG-I expression peaked at 
Table I. Characteristics of patients.

\begin{tabular}{lccccc}
\hline Groups & Age & Gender & $\begin{array}{c}\text { HBV DNA } \\
(\log 10 \text { copies } / \mathrm{ml})\end{array}$ & ALT & AST \\
\hline CHB & $39.47 \pm 2.84$ & $14 / 7$ & $6.74 \pm 0.24$ & $370.18 \pm 95.34$ & $217.94 \pm 64.91$ \\
AHB & $40.14 \pm 3.37$ & $4 / 3$ & $2.04 \pm 0.11$ & $1053.00 \pm 261.44$ & $550.00 \pm 186.02$ \\
HC & $37.67 \pm 2.71$ & $11 / 7$ & - & - & - \\
P-value & $0.843^{\mathrm{a}}$ & $0.594^{\mathrm{a}}$ & $<0.001$ & 0.005 & 0.043 \\
\hline
\end{tabular}

${ }^{a}$ The P-values for the age and gender between the 3 groups separately were (CHB vs. AHB 0.895, 0.938; CHB vs. HC 0.638, 0.337; AHB vs. HC 0.624, 0.526). HBV, hepatitis B virus; ALT, alanine aminotransferase; AST, aspartate aminotransferase; CHB, chronic hepatitis B; AHB, acute hepatitis B; HC, healthy control.

Table II. Sequences of primers used for PCR or qPCR of mRNA.

\begin{tabular}{lll}
\hline Gene name & \multicolumn{1}{c}{ Primer sequences $\left(5^{\prime} \rightarrow 3^{\prime}\right)$} \\
\hline RIG-I & Sense & TGTGCTCCTACAGGTTGTGGA \\
& Antisense & CACTGGGATCTGATTCGCAAAA \\
$I P S-1$ & Sense & CAGCAAGAGACCAGGATCGAC \\
& Antisense & CGTCCGCGAGATCAACTAGC \\
$I N F-\beta$ & Sense & GCGACACTGTTCGTGTTGTCA \\
& Antisense & CCAAGCAAGTTGTAGCTCATGGA \\
GAPDH & Sense & GAAGGTGAAGGTCGGAGTC \\
& Antisense & GAAGATGGTGATGGGATTTC \\
\hline
\end{tabular}

$R I G-I$, retinoic acid-inducible gene I; IPS-1, IFN- $\beta$ promoter stimulator $1 ; I F N-\beta$, interferon- $\beta$.

$16 \mathrm{~h}$ in the CHB, AHB and HC groups, at which point RIG-I expression was increased 9-, 27- and 17-fold relative to the unstimulated counterparts, respectively $(\mathrm{P}<0.01)$. A similar pattern was observed at $24 \mathrm{~h}$.

Expression of IPS-1 was induced in the moDCs of both AHB and CHB patients following VSV challenge, but not in the HCs (Fig. 2B). No significant induction was observed at $8 \mathrm{~h}$ post-challenge. However, at 16 and $24 \mathrm{~h}$ post-stimulation the level of IPS-1 induction in AHB moDCs was 1.1- and 2.9-fold higher than the unstimulated controls and the level of IPS-1 induction in CHB patients was 1.8- and 2.4-fold higher than the unstimulated controls.

Following VSV stimulation, IFN- $\beta$ expression was detected in the moDCs of AHB and CHB patients as well as $\mathrm{HCs}$ at various time points. There were no significant changes observed in the moDCs from CHB patients at various time points. However, IFN- $\beta$ expression in the moDCs from AHB patients was induced 3.3-, 5.8- and 5.6-fold at 8, 16 and $24 \mathrm{~h}$ compared with matched unstimulated moDCs (Fig. 2C). IFN- $\beta$ expression in the moDCs from HCs was 1.7-, 4.3- and 7.2-fold higher than unstimulated controls at 8,16 and $24 \mathrm{~h}$. Notably, IFN- $\beta$ expression in the moDCs from CHB patients at 16 and $24 \mathrm{~h}$ was significantly lower than from the other 2 groups $(\mathrm{P}<0.05)$.
A
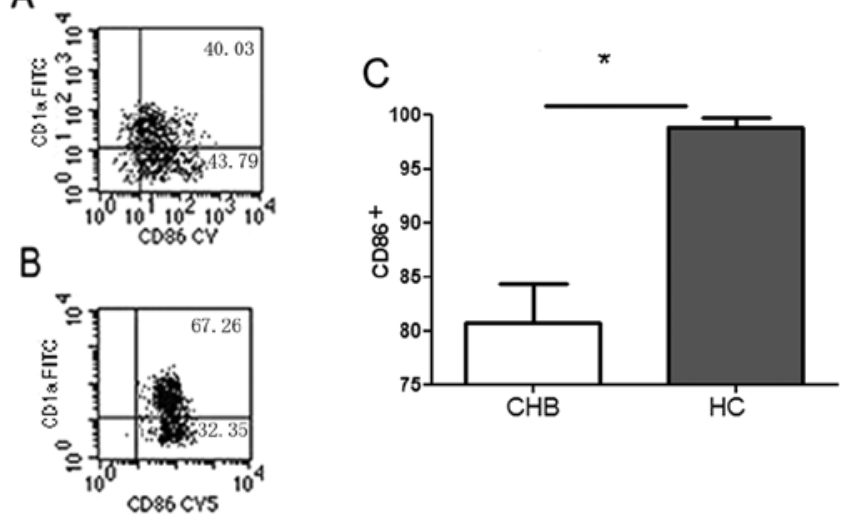

Figure 1. $\mathrm{CD} 86^{+}$moDCs were identified by flow cytometry in (A) CHB patients $(n=7)$, and $(B) \mathrm{HCs}(n=7)$. There was a $17 \%$ reduction of $\mathrm{CD} 86^{+}$ moDC in CHB compared with $\mathrm{HC}(80.77 \pm 8.51 \%$ vs. $96.89 \pm 2.12 \%)(\mathrm{P}<0.05)$. No data from AHB patients were available due to lack of samples. Data are presented as the mean \pm SD. ${ }^{*} \mathrm{P}<0.05$.

Correlation between RIG-I/IPS-1 and ALT, AST and HBV $D N A$. RIG-I and IPS-1 are involved in antiviral immunity, whereas alanine aminotransferase (ALT), aspartate aminotransferase (AST) and HBV DNA are important markers for liver injury and viral replication in HBV infection. The relationship among these factors is unclear. There was a significant correlation between ALT and RIG-I/IPS-1 in CHB patients $(\mathrm{P}<0.05)$ (Fig. 3A). No comparison was performed in AHB patients due to insufficient patient number. No significant correlation between AST and RIG-I/IPS-1 (Fig. 3B) or between HBV DNA and RIG-I/IPS-1 (Fig. 3C) was found in CHB patients.

RIG-I elevates IFN- $\beta$ secretion in HBV-transformed cells. RIG-I induces type-I interferon production, which also upregulates RIG-I expression during viral infection in a positive feedback loop (18). To determine the relationship between HBV infection and RIG-I production, the production of RIG-I and IFN- $\beta$ by RIG-I-transfected, HBV-transformed HepG2.2.15 cells was determined $24 \mathrm{~h}$ after VSV stimulation. There was a constant level of IFN- $\beta$ in the supernatant from both unstimulated and untransfected HepG2.2.15 and HepG2 cells (Fig. 4). There was a 5- or 6-fold elevation of IFN- $\beta$ production in the RIG-I-transfected HepG2.2.15 
A

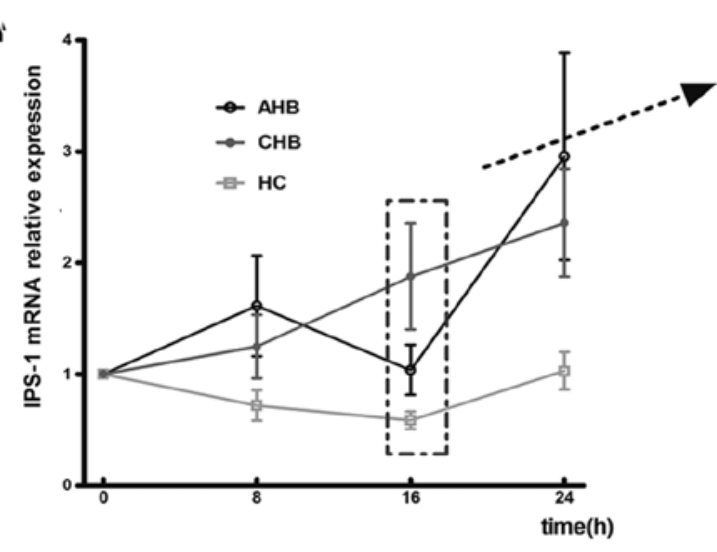

B

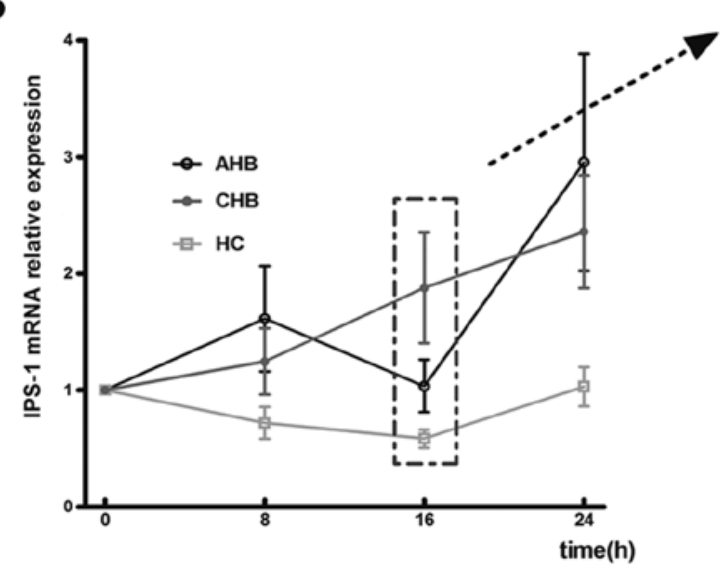

C

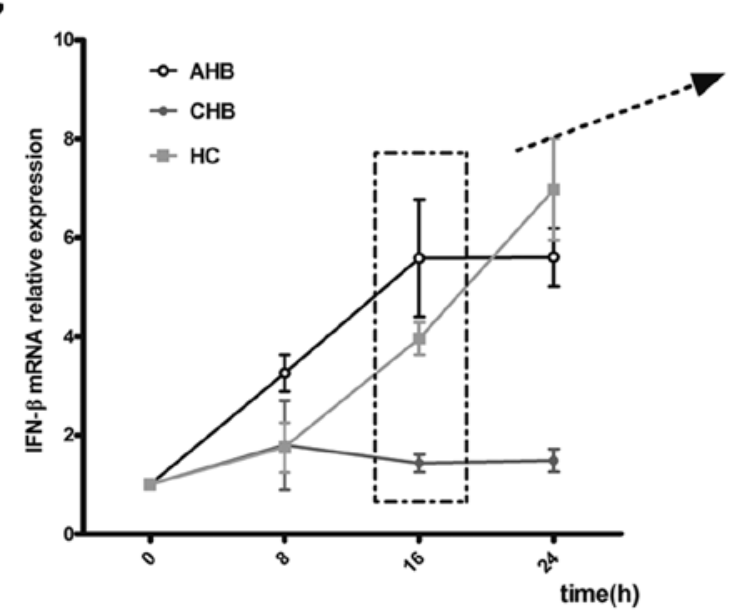

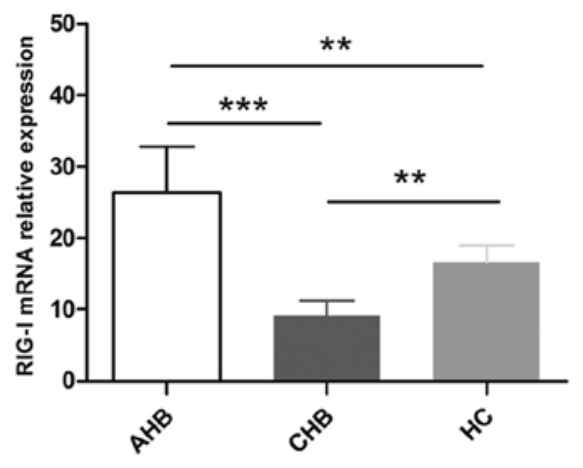
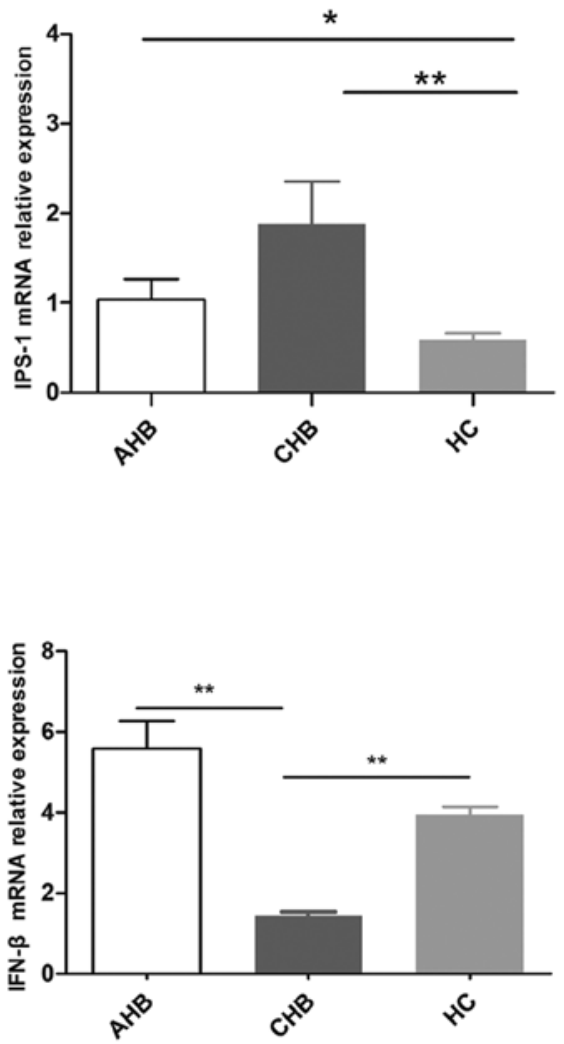

Figure 2. Expression of (A) RIG-I, (B) IPS-1 and (C) IFN- $\beta$ in the moDCs isolated from CHB, AHB and HC at different time points after VSV stimulation. The $\mathrm{x}$-axis represents the time after VSV stimulation (h) and the $\mathrm{y}$-axis represents the level of mRNA expression. Data are presented as the mean \pm SD. ${ }^{*} \mathrm{P}<0.05,{ }^{* *} \mathrm{P}<0.01,{ }^{* * * *} \mathrm{P}<0.001$.

cells regardless of VSV treatment. Markedly, there was an almost 100 -fold induction in IFN- $\beta$ production in the supernatant from RIG-I-transfected, VSV-stimulated cells. Following RIG-I transfection, IFN- $\beta$ production in HepG2 cells was $\sim 10$-fold higher than in HepG2.2.15 cells $(\mathrm{P}<0.01)$, whereas more profound IFN- $\beta$ production was only observed following VSV challenge. There was a $~ 500$-fold induction of IFN- $\beta$ production in both HepG2.2.15 and HepG2 cells in response to combined RIG-1 transfection and VSV challenge compared with the mock-treated counterparts. However, no significant difference was observed between the HepG2.2.15 and HepG2 cells.

\section{Discussion}

HBV is a member of the Hepadnaviridae family, which consists of hepatotropic non-cytopathic DNA viruses. Host immunity plays a critical role in determining the outcome following HBV infection (19). Mature dendritic cells (DC) are competent antigen-presenting cells that play key roles 

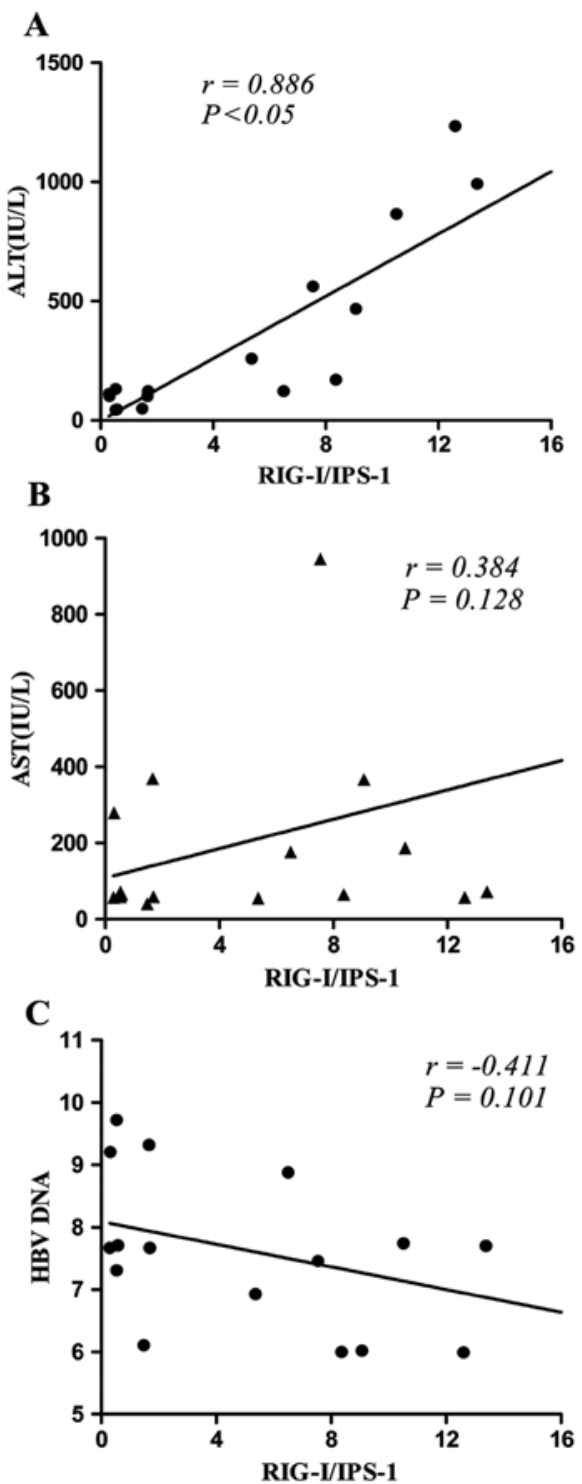

Figure 3. Correlation between (A) RIG-I/IPS-1 and ALT, (B) RIG-I/IPS-1 and AST, and (C) RIG-I/IPS-1 and HBV DNA was determined. Data are presented as the mean $\pm \mathrm{SD}$.

in priming and directing the viral-specific T-cell response. Dendritic cells induce inflammation following challenge from an invading microorganism, a critical step in the initiation of the innate immune response. Due to difficulty in obtaining mature DCs from peripheral circulation in humans ( $<1 \%$ of human PBMCs) (14), moDCs were generated in vitro as described (20). Our data demonstrated that the proportion of $\mathrm{CD}^{2} 6^{+}$mature moDCs was significantly reduced in CHB patients compared with HCs following VSV stimulation, suggesting that chronic HBV infection blocks the maturation of moDCs and subsequently compromises the activation of specific anti-HBV immunity in CHB patients $(21,22)$.

Activation of RIG-I by viral RNA stimulation results in the activation of the IFN system and thus plays a pivotal role in the antiviral response. However, in susceptible individuals, viruses can still circumvent the IFN response through unknown mechanisms (23), resulting in chronic viral infection.

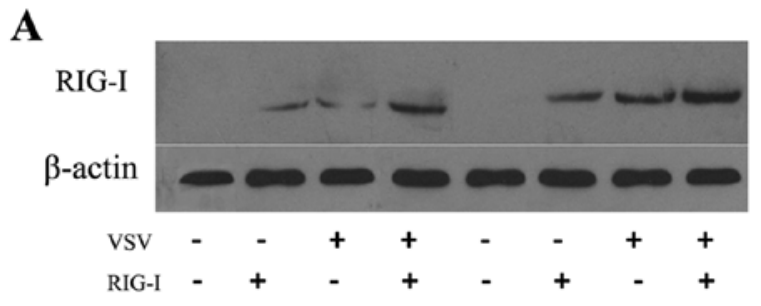

B

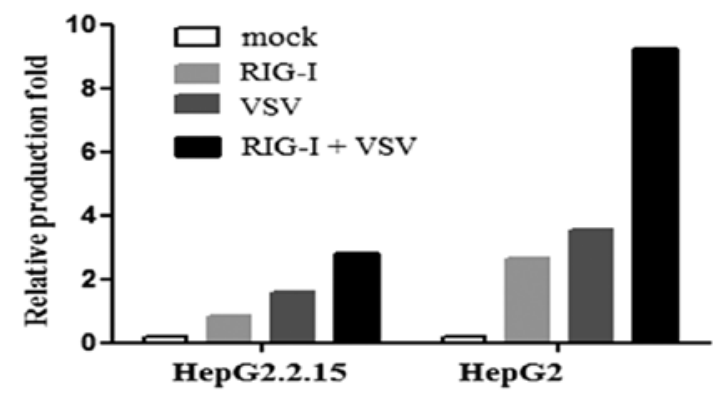

C

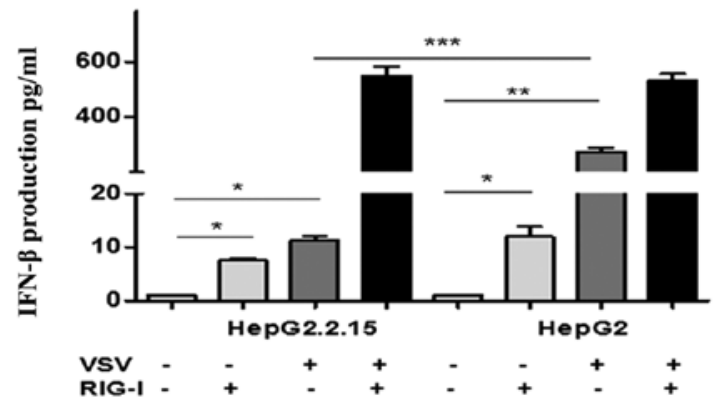

Figure 4. RIG-I levels in the HepG2 and HepG2.2.15 cell lines were (A) detected by western blot analysis, and (B) quantified using image analysis software. (C) IFN- $\beta$ was detected in the supernatant from HepG2 and HepG2.2.15 cell lines \pm RIG-I transfection and VSV stimulation by ELISA. The $\mathrm{x}$-axis represents different treatments and the $\mathrm{y}$-axis represents the level of IFN- $\beta$. Data are presented as the mean $\pm \mathrm{SD} .{ }^{*} \mathrm{P}<0.05,{ }^{* *} \mathrm{P}<0.01$, ${ }^{* * *} \mathrm{P}<0.001$.

Here, we demonstrated that the induction of RIG-I and IFN- $\beta$ expression was compromised in moDCs isolated from CHB patients compared with both AHB and HC subjects following VSV stimulation in vitro, suggesting that the RIG-I-IFN pathway was disrupted in the CHB patients. Consistent with these findings, there was also a $90 \%$ reduction in IFN- $\beta$ production in HBV-transformed HepG2.2.15 cells relative to HepG2 cells following VSV stimulation, which further indicated that HBV interferes with IFN- $\beta$ production. Compromised IFN- $\beta$ production was restored in HepG2.2.15 cells by RIG-1 overexpression, demonstrating the critical role of RIG-I in the production of IFN- $\beta$ in response to HBV infection. The mechanisms underlying the differences in RIG-I and IFN- $\beta$ production among CHB and AHB patients remain unclear, but may involve the complicated host anti-HBV immunity and/or differential regulation of other receptors (such as TLRs) and pathways at various stages: immune tolerance phase, immune clearance phase and inactive phase (19). 
The different host immunities in $\mathrm{CHB}$ and AHB patients may also be due to the immune response against AHB infection being polyclonal, multi-specific and non-tolerant, that is, the host immune system adopts a highly sensitive status (24), whereas in the chronic stage, host immunity is immunetolerant. This hypothesis is supported by the finding that more IFN- $\beta$ was produced in the moDCs from AHB patients following VSV infection, which provides positive feedback to boost RIG-I expression. Increased IFN- $\beta$ production may also explain why RIG-I levels in AHB moDCs were the highest at $16 \mathrm{~h}$ (representing a highly sensitive status). This observation is in line with the findings that IFN- $\beta$ production in AHB patients was higher than in $\mathrm{CHB}$ patients and suggests that an impaired RIG-I pathway contributes to compromised IFN- $\beta$ production in $\mathrm{CHB}$ patients.

It has been reported that the RNA virus directly downregulates RIG-I and its downstream adaptor IPS-1 (11), resulting in the inhibition of IFN- $\beta$ production. In the current study, IPS-1 expression in moDCs from $\mathrm{CHB}$ patients was significantly higher than that of HCs and was inversely correlated with IFN- $\beta$ production. The high IPS-1 level in moDCs from CHB patients may be due to compensation for compromised IPS-1 function and subsequent induction of IFN- $\beta$ production. The underlying mechanisms leading to compromised IPS-1 function remain to be explored, but interference from HBV may be a contributing factor, as Lin et al (25) have reported that the HCV protease NS3-4A degrades the IPS-1 protein.

To investigate the relationship between liver damage (ALT) and immune regulation (RIG-1 signaling), the correlation between ALT level and the ratio of RIG-I to IPS-1 (RIG-I/IPS-1) was determined (26). The correlation between RIG-I and IPS-1 levels was also evaluated as a measure of RIG-I-IFN- $\beta$ signaling pathway activity. We observed a positive correlation between ALT level and RIG-I/IPS-1, indicating that activation of RIG-I signaling by HBV infection in moDCs may induce liver damage. The RIG-I signaling pathway activates antiviral responses via IFN- $\beta$ to clear invaded viruses and/or infected hepatocytes. Liver damage in CHB patients with high ALT levels may also result from other activated host lymphocytes, such as HBV-specific cytotoxic lymphocyte (CTL) and natural killer (NK) T cells $(27,28)$.

Neither AST nor HBV DNA is a reliable indicator of inflammation in CHB (29). Our data showed no correlation between AST and the RIG-I/IPS-1 ratio or between HBV DNA and the RIG-I/IPS-1 ratio in CHB patients, suggesting that further investigation is required to elucidate the relationship between AST, HBV DNA and RIG-I/IPS-1.

In summary, compromised moDC maturation and function in CHB patients may result from a dysfunctional RIG-I-IFN- $\beta$ signaling pathway and consequent impairment of host viral clearance, which we propose are contributing factors to HBV persistence in a susceptible population.

\section{Acknowledgements}

This study was supported by the Natural Science Foundation of China (30671838, 81070334); the National Eleven-Five Project of China (2008ZX10002-005, 2008ZX10002-007); and the Committee of Science and Technology of Shanghai Municipal Government (08JC1403900).

\section{References}

1. Lok AS and McMahon BJ: Chronic hepatitis B: update 2009. Hepatology 50: 661-662, 2009.

2. Marinos G, Torre F, Chokshi S, et al: Induction of T-helper cell response to hepatitis $\mathrm{B}$ core antigen in chronic hepatitis $\mathrm{B}$ : a major factor in activation of the host immune response to the hepatitis B virus. Hepatology 22: 1040-1049, 1995.

3. Hatakeyama T, Noguchi C, Hiraga N, et al: Serum HBV RNA is a predictor of early emergence of the YMDD mutant in patients treated with lamivudine. Hepatology 45: 1179-1186, 2007.

4. Cheng G, Zhong J, Chung J and Chisari FV: Double-stranded DNA and double-stranded RNA induce a common antiviral signaling pathway in human cells. Proc Natl Acad Sci USA 104: 9035-9040, 2007.

5. Lee MS and Kim YJ: Signaling pathways downstream of pattern-recognition receptors and their cross talk. Annu Rev Biochem 76: 447-480, 2007.

6. Hornung V, Ellegast J, Kim S, et al: 5'-Triphosphate RNA is the ligand for RIG-I. Science 314: 994-997, 2006.

7. Yoneyama M, Kikuchi M, Natsukawa T, et al: The RNA helicase RIG-I has an essential function in double-stranded RNA-induced innate antiviral responses. Nat Immunol 5: 730-737, 2004.

8. Kato H, Sato S, Yoneyama M, et al: Cell type-specific involvement of RIG-I in antiviral response. Immunity 23: 19-28, 2005.

9. Fujita T, Onoguchi K, Onomoto K, Hirai R and Yoneyama M: Triggering antiviral response by RIG-I-related RNA helicases. Biochimie 89: 754-760, 2007.

10. Binder M, Kochs G, Bartenschlager R and Lohmann V: Hepatitis $C$ virus escape from the interferon regulatory factor 3 pathway by a passive and active evasion strategy. Hepatology 46: 1365-1374, 2007.

11. Komuro A, Bamming D and Horvath CM: Negative regulation of cytoplasmic RNA-mediated antiviral signaling. Cytokine 43: 350-358, 2008

12. Cai W, Xie Q, An B, et al: On-treatment serum HBsAg level is predictive of sustained off-treatment virologic response to telbivudine in $\mathrm{HBe} \mathrm{Ag}$-positive chronic hepatitis B patients. J Clin Virol 48: 22-26, 2010.

13. Lin LY, Wong VW, Zhou HJ, et al: Relationship between serum hepatitis B virus DNA and surface antigen with covalently closed circular DNA in HBeAg-negative patients. J Med Virol 82: 1494-1500, 2010.

14. Dauer M, Obermaier B, Herten J, et al: Mature dendritic cells derived from human monocytes within 48 hours: a novel strategy for dendritic cell differentiation from blood precursors. J Immunol 170: 4069-4076, 2003.

15. Nakhaei P, Mesplede T, Solis M, et al: The E3 ubiquitin ligase Triad3A negatively regulates the RIG-I/MAVS signaling pathway by targeting TRAF3 for degradation. PLoS Pathog 5: e1000650, 2009.

16. Sun D and Nassal M: Stable HepG2- and Huh7-based human hepatoma cell lines for efficient regulated expression of infectious hepatitis B virus. J Hepatol 45: 636-645, 2006.

17. tenOever BR, Sharma S, Zou W, et al: Activation of TBK1 and IKKvarepsilon kinases by vesicular stomatitis virus infection and the role of viral ribonucleoprotein in the development of interferon antiviral immunity. J Virol 78: 10636-10649, 2004.

18. Huang Q, Xie Q, Shi CC, et al: Expression of angiotensinconverting enzyme 2 in CCL4-induced rat liver fibrosis. Int $\mathrm{J}$ Mol Med 23: 717-723, 2009.

19. Jung MC and Pape GR: Immunology of hepatitis B infection. Lancet Infect Dis 2: 43-50, 2002.

20. Terhorst D, Kalali BN, Weidinger S, et al: Monocyte-derived dendritic cells from highly atopic individuals are not impaired in their pro-inflammatory response to toll-like receptor ligands. Clin Exp Allergy 37: 381-390, 2007.

21. Patterson S, Donaghy H, Amjadi P, Gazzard B, Gotch F and Kelleher P: Human BDCA-1-positive blood dendritic cells differentiate into phenotypically distinct immature and mature populations in the absence of exogenous maturational stimuli: differentiation failure in HIV infection. J Immunol 174: 8200-8209, 2005.

22. Shimizu Y, Guidotti LG, Fowler P and Chisari FV: Dendritic cell immunization breaks cytotoxic $\mathrm{T}$ lymphocyte tolerance in hepatitis B virus transgenic mice. J Immunol 161: 4520-4529, 1998. 
23. Randall RE and Goodbourn S: Interferons and viruses: an interplay between induction, signalling, antiviral responses and virus countermeasures. J Gen Virol 89: 1-47, 2008.

24. Hui CK and Lau GK: Immune system and hepatitis B virus infection. J Clin Virol 34: S44-S48, 2005.

25. Lin R, Lacoste J, Nakhaei P, et al: Dissociation of a MAVS/ IPS-1/VISA/Cardif-IKKepsilon molecular complex from the mitochondrial outer membrane by hepatitis C virus NS3-4A proteolytic cleavage. J Virol 80: 6072-6083, 2006.

26. Asahina Y, Izumi N, Hirayama I, et al: Potential relevance of cytoplasmic viral sensors and related regulators involving innate immunity in antiviral response. Gastroenterology 134: 1396-1405, 2008.
27. Stoop JN, van der Molen RG, Baan CC, et al: Regulatory T cells contribute to the impaired immune response in patients with chronic hepatitis B virus infection. Hepatology 41: 771-778, 2005.

28. Zerbini A, Pilli M, Boni C, et al: The characteristics of the cellmediated immune response identify different profiles of occult hepatitis B virus infection. Gastroenterology 134: 1470-1481, 2008.

29. Chang JJ and Lewin SR: Immunopathogenesis of hepatitis B virus infection. Immunol Cell Biol 85: 16-23, 2007. 